\title{
Initial and Post-Initial Acquisition in the Serial Search Based Noncoherent Multiple Transmit/Receive Antenna Aided DS-CDMA Downlink
}

\author{
SeungHwan Won and Lajos Hanzo \\ School of ECS, Univ. of Southampton, SO17 1BJ, UK. \\ Tel: +44-23-8059 3125, Fax: +44-23-8059 4508 \\ E-mail:\{shw04r, lh\}@ecs.soton.ac.uk \\ http://www-mobile.ecs.soton.ac.uk
}

\begin{abstract}
In this paper we investigate the issues of both initial and post-initial acquisition schemes in the multiple transmit/receive antenna aided DS-CDMA downlink, when communicating over uncorrelated Rayleigh channels. The associated Mean Acquisition Time (MAT) performance trends are characterised as a function of the number of transmit/receive antennas. Furthermore, we characterise both the initial and post-initial acquisition performance as a function of the relevant system parameters. It is demonstrated that in contrast to our expectations, the achievable MAT degrades at low $E_{c} / I_{o}$ values, except for the case of $P=2$ transmit antennas operating in conjunction with $R=1$ receive antenna over the specific Signal-to-Interference plus Noise Ratio (SINR) per chip $\left(E_{c} / I_{o}\right)$ range considered, as the number of transmit antennas is increased. Ironically, our findings suggest that increasing the number of transmit antennas in a MIMO-aided CDMA system results in combining the low-energy, noise-contaminated signals of the transmit antennas, which ultimately increases the MAT by an order of magnitude, when the SINR is relatively low. This phenomenon has a detrimental effect on the performance of Rake receiver based synchronisation schemes, when the perfectly synchronised system is capable of attaining its target bit error rate performance at reduced SINR values, as a benefit of employing multiple transmit antennas. Therefore our future research will be focused on specifically designing acquisition schemes for MIMO systems.
\end{abstract}

\section{INTRODUCTION}

A variety of schemes employing multiple antennas in the downlink of wireless systems constitute an attractive technique of reducing the detrimental effects of time-variant multi-path fading environments [1]. In inter-cell synchronous CDMA systems the mobile station's (MS) receiver must be capable of perfectly synchronously aligning a locally generated pseudonoise (PN) code with the received multiuser signals containing the desired user's PN sequence. Substantial research efforts have been devoted to the design of code acquisition techniques [2],[3]. However, most of these acquisition schemes have been designed for Singe-Input Single-Output (SISO) systems, with the aim of optimising the achievable Mean Acquisition Time (MAT) performance. By contrast, there is a paucity of code acquisition techniques designed for optimum post-initial acquisition performance [4], [5], where the term 'post-initial acquisition [4]' refers to identifying the timing instants of the affordable-complexity-dependent number of delayed received signal paths, which will be combined by Rake receiver. Moreover, since there are no in-depth studies representing the fundamental characteristics of code acquisition schemes assisted by multiple transmit/receive antennas in the context of both initial and post-initial acquisition schemes, this is the objective of the present contribution. Against this background, in this treatise we investigate serial search based noncoherent code acquisition schemes designed for multiple transmit/receive antenna aided systems for the sake of analysing the characterisitics of both initial and post-initial acquisition schemes. More explicitly, we quantify both the correct detection probability as well as the false alarm probability as a function of both the SINR per chip and that of the number of transmit/receive antennas. Furthermore, we characterise the attainable MAT versus $E_{c} / I_{o}$ performance parameterised by the number of transmit/receive antennas. This paper is organised as follows. The MAT analysis of both initial and post-initial acquisition is illustrated in Section II, while Section III describes the system investigated, followed by the correct detection and false alarm probability analysis of noncoherent code acquisition schemes in the context of uncorrelated Rayleigh channels. In Section IV, our numerical MAT results are discussed, while our conclusions are offered in Section V.

\section{MAT ANALYSIS OF INITIAL AND POST-INITIAL ACQUISITION}

The classic serial search techniques designed for intial acquisition [6] have been traditionally employed in specific scenarios, where the uncertainty region (or search window width) is quite wide (i.e. $2^{15}-1$ ) and hence in the context of serial search the MAT constitutes the vital performance criterion, as seen for example in the downlink of the inter-cell synchronous CDMA-2000 system [6]. In the case of initial acquisition contrived for DS-CDMA, the main design goal is to acquire perfect timing of the first received signal path impinging at the receiver, since this timing information is used as that of the reference finger of the Rake receiver. By contrast, the post-initial acquisition procedure that extracts the accurate timing positions of the remaining delayed paths and identifies the appropriate paths earmarked for processing by the Maximum Ratio Combining (MRC) scheme of the Rake receiver, has a major impact on the performance of the Rake receiver [5]. There are two main differences between the initial and post-initial acquisition procedures. First of all, once the first Rake finger is synchronised, the uncertainty region that has to be explored will be shrunk to $\pm U$ hypotheses surrounding the time-instant, where the first received path was found. This reduced interval will be referred as to the compressed uncertainty region [4]. This search window width is defined by both the dispersion of the multipath propagation environment encountered as well as by the appearance and disappearance of propagation paths [7]. Secondly, the post-initial acquisition procedure commences after Automatic Frequency Control (AFC) operation was activated for the sake of fine tracking, following the successful initial acquisition. Hence, the performance degradation imposed by the associated frequency mismath is considerably reduced compared to that immediately after the initial acquisition. Accordingly, these two factors are taken into account in our forthcoming analysis.

In [6],[8], explicit MAT formulae were provided for a singleantenna aided serial search based code acquisition system. There is no distinction between a single-antenna aided scheme and a multipleantenna assisted one in terms of analysing the MAT, except for 
deriving the correct detection and the false alarm probability based upon the multiple transmit/receive antennas. We will commence our discourse by analysing the MAT performance of both initial and post-initial acquisition schemes employing both Single Dwell Serial Search (SDSS) [6] as well as Double Dwell Serial Search (DDSS) [8]. We assume that in each chip duration $T_{c}, l$ number of correct timing hypotheses are tested, which are spaced by $T_{c} / l$. Hence the total uncertainty region is increased by a factor of $l$. All the resultant $(\nu-2 l)$ states that may lead to a false alarm are expected to increase the MAT according to the corresponding penalty time. The $2 l$ legitimate locking states within a lag of one chip duration of the correct timing instant are taken into account in the MAT analysis. The requried transfer functions [6],[8], are defined as follows. The function $H_{D}(z)$ encompasses all branches of a state diagram [6], [8] leading to successful detection, $H_{0}(z)$ indicates the absence of the desired user's signal at the output of the acquisition scheme, while $H_{M}(z)$ represents the overall miss probability of a search run carried out across the entire uncertainty region, all of which are detailed in [6] for both SDSS [6] and for DDSS [8]. Then, it may be shown that the generalised expression derived for computing the MAT of the serial search based code acquisition scheme is given by [6]:

$$
\begin{array}{r}
E\left[T_{A C Q}\right]=\frac{1}{H_{D}(1)}\left[H_{D}{ }^{\prime}(1)+H_{M}{ }^{\prime}(1)+\right. \\
\left.\left\{(\nu-2 l)\left[1-\frac{H_{D}(1)}{2}\right]+\frac{1}{2} H_{D}(1)\right\} H_{0}{ }^{\prime}(1)\right] \cdot \tau_{D 1},
\end{array}
$$

where $\left.H_{x}^{\prime}(z)\right|_{x=D, M \text { or } 0}$ is a derivative of $\left.H_{x}(z)\right|_{x=D, M \text { or } 0}$ and $\tau_{D 1}$ denotes the $1^{\text {st }}$ dwell time.

\section{CORRECT DETECTION AND FALSE ALARM PROBABILITY}

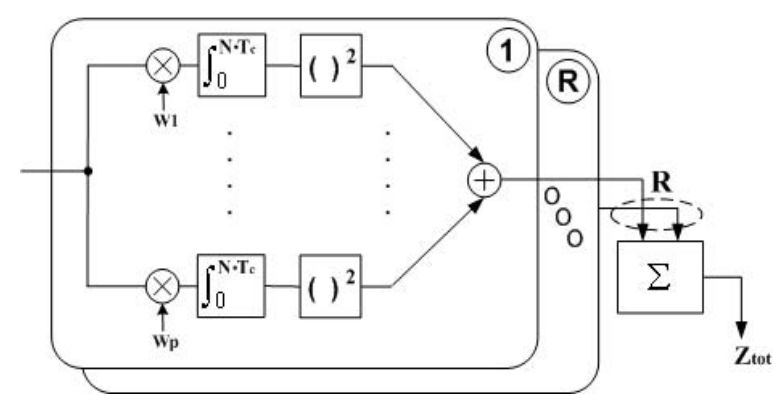

Fig. 1. Receiver structure of a noncoherent code acquisition system employing $R$ receive antennas. Its basic operation is identical for both the initial and post-initial acquisition schemes except for using different coherent summation intervals necessitated by the different frequency mismatch of the two schemes

The received signal of the MIMO-aided noncoherent DS-CDMA downlink may be expressed as

$$
\begin{array}{r}
r(t)=\sum_{m=1}^{P} \sum_{n=1}^{R}\left[\alpha_{m, n} \sqrt{\frac{E_{c}}{P T_{c}}} c\left(t+d T_{c}\right) w_{m}\left(t+d T_{c}\right)\right. \\
\left.\cdot \exp \left(2 \pi f t+\phi_{m, n}\right)+I_{k(m, n)}(t)\right],
\end{array}
$$

where $m=1, \ldots, P$ is the number of transmit antennas, $n=1, \ldots, R$ is the number of receive antennas, $\alpha_{m, n}$ represents the envelope of the $(m, n)^{t h}$ path signal obeying the Rayleigh distribution, $E_{c}$ denotes the pilot signal energy per PN code chip, $c(t)$ represents a unique user-specific PN sequence, $d$ is the code phase offset with respect to the phase of the local code, $T_{c}$ is the chip duration, $w_{m}(t)$ identifies the specific Walsh code assigned to the $m^{t h}$ transmit antenna, $f$ is the carrier frequency and finally, $\phi$ is the carrier phase of a specific user's modulator. Furthermore, $I_{k(m, n)}(t)$ is the complex-valued additive white Gaussian (AWGN) noise having a double-sided power spectral density of $I_{0}$ at the $(m, n)^{t h}$ path. Here the total allocated power is equally shared by the $P$ transmit antennas. Fig. 1 depicts the block diagram of the noncoherent receiver designed for our code acquisition scheme using multiple transmit/receive antennas, which generates a decision variable by accumulating $(P \cdot R)$ number of independently faded signals observed over a time interval for the sake of improving the correct detection probability in the mobile channel imposing both fading and poor SINR conditions.

By employing the procedures proposed in [9] in the context of the receiver structure of Fig.1, the final decision variable may be written as

$$
Z_{k}=\sum_{m=1}^{P} \sum_{n=1}^{R}\left\|\frac{1}{\sqrt{2}} \cdot\left(\sqrt{\frac{4 E_{c}}{N I_{0} P}} \cdot S_{k(m, n)}+I_{k(m, n)}\right)\right\|^{2},
$$

where $k$ denotes the $k^{t h}$ chip's sampling instant, $S_{k(m, n)}$ is assumed to be deterministic as described in [9], $\|\cdot\|^{2}$ represents the squared envelope of the complex-valued argument and the factor of $1 / \sqrt{2}$ is employed to normalise the noise variance. Accordingly, the decision variable $Z_{k}$ obeys a noncentral chi-square PDF with $(2 P \cdot R)$ degrees of freedom and its noncentrality parameter $\lambda_{x}$ is either $\frac{2 N}{P}\left(\frac{E_{c}}{I_{0}}\right)$ for the hypothesis of the desired signal being present $(x=1)$ or $\frac{2}{N P}\left(\frac{E_{c}}{I_{0}}\right)$ for it being absent $(x=0)$ [9], where the PDF is given by [10]

$$
\begin{array}{r}
f_{Z_{k}}\left(z \mid H_{x}\right)=\frac{1}{2}\left(\frac{z}{\lambda_{x}}\right)^{\frac{(P R-1)}{2}} \cdot \exp \left[-\frac{\left(z+\lambda_{x}\right)}{2}\right] \\
\cdot \mathcal{I}_{(P R-1)}\left(\sqrt{z \cdot \lambda_{x}}\right)
\end{array}
$$

and where $z \geq 0, x=0$ or 1 and $\mathcal{I}_{(P R-1)}(\cdot)$ is the $(P \cdot R-1)^{s t}$ order modified Bessel function. Our aim is now that of expressing the PDF of a desired user's signal at the output of the acquisition scheme conditioned on the presence of the desired signal in $f_{Z_{k}}\left(z \mid H_{x}\right)$ derived for transmission over an uncorrelated Rayleigh channel. Hence first the PDF $f_{Z_{k(m, n)}}\left(z \mid H_{x}, \beta\right)$ corresponding to a specific SINR $\beta$ conditioned on the hypothesis of the desired signal being transmitted over an AWGN channel having this specific SINR is weighted by the probability of occurrence $f(\beta)$ of encountering the SINR $\beta$, as quantified by the PDF. The resultant product is then averaged over its legitimate range of $-\infty \sim \infty$, yielding:

$$
\begin{array}{r}
f_{Z_{k(m, n)}}\left(z \mid H_{x}\right)=\int_{-\infty}^{\infty} f(\beta) \cdot f_{Z_{k(m, n)}}\left(z \mid H_{x}, \beta\right) d \beta \\
=\int_{0}^{\infty}\left(\frac{e^{-\beta / \sigma^{2}}}{\sigma^{2}}\right) \cdot \frac{\exp \left[-\left(z+\beta \lambda_{x}\right) / 2\right]}{2} \\
\\
=\frac{\mathcal{I}_{0}\left(\frac{2 \sqrt{\beta \lambda_{x} z}}{2}\right) d \beta}{\left(2+\lambda_{x} \sigma^{2}\right)} \\
\equiv \frac{\exp \left[-z /\left(2+\lambda_{x} \sigma^{2}\right)\right]}{\left.\left(2+\overline{\lambda_{x}}\right)\right]}
\end{array}
$$


where the effects of both timing errors and frequency mismatches are encapsulated by the definition of $\left(E_{c} / I_{0}\right)^{\prime}$ [6] and the corresponding noncentrality parameter, $\overline{\lambda_{x}} \equiv \lambda_{x} \sigma^{2}$ is either $\frac{2 N}{P}\left(\overline{\frac{E_{c}}{I_{0}}}\right)^{\prime}$ when the desired signal is deemed to be present $(x=1)$ or $\frac{2}{N P}\left(\frac{\overline{E_{c}}}{I_{0}}\right)^{\prime}$ when it is deemed to be absent $(x=0)$. We also define $\mu_{x}=\left(2+\overline{\lambda_{x}}\right)$, which physically represents a new biased noncentrality parameter. Further details on the related calculations can be found in [6], [9]. Finally, we arrive at the PDF of $Z_{k(m, n)}$ conditioned on the presence of the desired signal in the form of:

$$
f_{Z_{k(m, n)}}\left(z \mid H_{x}\right)=\frac{1}{\mu_{x}} e^{-z / \mu_{x}} .
$$

Since the decision variables $Z_{k}$ is constituted by the sum of $(P \cdot R)$ number of independent variables $\left(Z_{k}=\sum_{m=1}^{P} \sum_{n=1}^{R} Z_{k(m, n)}\right)$, which has a PDF given by Eq (9), we can determine the Laplace transform of each by raising them to the $(P \cdot R)^{t h}$ power and then carrying out the inverse transform for the sake of generating the desired PDF [6], leading to:

$$
f_{Z_{k}}\left(z \mid H_{x}\right)=\frac{z^{(P R-1)} e^{-z / \mu_{x}}}{\Gamma(P R) \cdot \mu_{x}^{P R}},
$$

where $\Gamma(\cdot)$ is the Gamma function. Finally, the probability of correct detection or false alarm corresponding to $x=1$ or 0 , respectively, is obtained as

$$
\begin{aligned}
\left.P\right|_{x=1 \text { or } 0} & =\int_{\theta}^{\infty} f_{Z_{k}}\left(z \mid H_{x}\right) d z \\
& =\exp \left(-\frac{\theta}{\mu_{x}}\right) \cdot \sum_{k=0}^{P R-1} \frac{\left(\theta / \mu_{x}\right)^{k}}{k !},
\end{aligned}
$$

where $\theta$ is a threshold value. By employing Eq (12), the transfer functions required for the multiple antenna aided scheme may be derived.

\section{NUMERICAL SYSTEM PERFORMANCE RESULTS}

In this section we will characterise the MAT performance of the multiple transmit/receive antenna aided DS-CDMA code acquisition scheme of Fig.1. In Table 1 and 2 we outlined the maximum SINR degradation imposed by both the Doppler shift and the frequency mismatch between the transmitter and receiver in conjunction with the coherent integration interval of $N$ chip durations seen in Fig.1 for both initial and post-initial acquisition. The length of the PN sequence in our system was assumed to be $\left(2^{15}-1\right) \cdot T_{c}$, where the chipduration is $T_{c}=1 / 1.2288 \mu \mathrm{s}$. In the case of the initial acquisition scheme of Fig.1, it was found to be sufficient to integrate the detector output seen in Fig. 1 over $N=128$ chips for the sake of analysing SDSS, while the number of chips over which the accumulator $\Sigma$ of Fig. 1 sums the $(\cdot)^{2}$ envelope detector's output in both the search and the verification modes of DDSS are assumed to be 32 and 256 in the $R=1$ and 2 receive antennas scenarios or 128 in the $R=$ 4 receive antenna senario, respectively. By contrast, in the case of the post-initial acquisition scheme, the optimised length of coherent summation of the detector output values invoked for the sake of analysing SDSS is given in Table 3, whilst 64 is selected as the length of coherent summation in the search mode of DDSS. Finally, the optimised intervals of the coherent summation used in the verification mode of DDSS are portrayed in Table 4. In both Table 3 and 4, the numbers seen in $(\cdot)$ can be used as an altenative. These optimised parameter values were calculated by using both Eq (1) and Eq (12) as well as the appropriate equations of [6] provided for determining the performance degradation owing to both the Doppler shift and the frequency mismatch. The spreading factor of the Walsh code to be acquired was selected to be 128. The frequency mismatch was assumed to be $1000 \mathrm{~Hz}$ for the initial acquisition [6] and $200 \mathrm{~Hz}$ for the post-initial acquisition phases [5], while the carrier frequency was $1.9 \mathrm{GHz}$. As a worst-case mobile speed, it is reasonable to postulate $160 \mathrm{~km} / \mathrm{h}$. We also assumed that the sampling inaccuracy caused by having a finite, rather than infinitesimally low search step size of $\Delta=1 / 2 T_{c}$ was $-0.91 \mathrm{~dB}$, which is a typical value for the search step size [6]. The total uncertainty region of initial and postinitial acquisition were assumed to entail 65534 and 124 hypotheses, respectively. Finally, in the spirit of [11], the false locking penalty factor was assumed to be 1000. For simplicity, it was assumed that only a single received signal path is encountered in a given search window. All the performance curves have been obtained at the optimum decision threshold of $E_{c} / I_{o}=-13 d B$ designed for the initial acquisition scheme and at $E_{c} / I_{o}=-19 d B$ invoked for the post-initial acquisition scheme, respectively. Operational range of the post-initial acquisition scheme was assumed to be $6 \mathrm{~dB}$ lower than that of the initial acquisition arrangement because the signal power of the delayed paths is typically lower than that of the first received path.

Fig.2 illustrates the achievable MAT versus SINR per chip performance for SDSS of the initial acquisition scheme as a function of the number of transmit antennas for $P=1,2$ as well as 4 and that of the number of receive antennas for $R=1,2$ and 4 . Observe in Fig.2 that surprisingly, as the number of transmit antennas is decreased, despite the potentially reduced transmit diversity gain, we experience an improved MAT performance. For comparison, Fig. 3 characterises the MAT versus SINR per chip performance of DDSS for the initial acquisition arrangement as a function of the number of transmit antennas for $P=1,2$ as well as 4 and that of the number of receive antennas for $R=1,2$ and 4 . Similarly to the conclusions of Fig.2, as the number of transmit antennas is decreased, all the curves seen in Fig. 3 illustrate an improved MAT peformance, except for the 'P2R1' scenario over the specific SINR range between -4 and $-11 \mathrm{~dB}$. To illustrate the above fact a little further, in the case of ' $P 2 R 1^{\prime}$ the DDSS scheme exhibits a better MAT peformance in comparison to the ' $P 1 R 1^{\prime}$ arrangement across the specific SINR range shown in Fig.3. This is because in the case of DDSS the reliable operational ranges in terms of both the correct detection and the false alarm probability are quite different from those of SDSS. More explicitly, the reliable operational range of SDSS associated with the best possible MAT performance is around a false alarm probability of $10^{-4}$. On the other hand, the reliable operation of DDSS may be maintained at as high a false alarm probability, as 0.2 when the number of transmit antennas is increased from $P=1$ to $P=4$ in conjunction with $R=1$ receive antenna, as demonstrated in Fig.4. Furthermore, in case of $R=4$ receive antennas, similar trends are observed, even though the region of the reliable DDSS operation is shifted to the left with respect to the case of a single receive antenna, as seen in Fig.4. It is worth mentioning that the operating range of $R=2$ receive antennas is in between that corresponding to $R$ $=1$ and $R=4$ receive antennas, for the sake of avoiding obfuscating points in the figure, the $R=2$ scenario was omitted. Accordingly, while the reliable operational range of SDSS is around a false alarm probability of $10^{-4}$, that of DDSS in the search mode varies more 
widely, namely across the range spanning from 0.04 to just over 0.2 , depending on the specific number of transmit and receive antennas. This manifests itself also in terms of having detection threshold values in the search mode of DDSS, which are substantially lower than those of SDSS, when optimised for the sake of attaining the best possible MAT performance. This clearly implies that DDSS benefits from a significantly higher diversity gain than SDSS. The peformance degradation imposed by employing multiple antennas becomes more drastic, as the number of transmit antennas is increased for both the SDSS and DDSS schemes in the initial acquisition scenario. Furthermore, the associated MAT performance discrepancy between the SDSS and DDSS schemes becomes more drastic. In case of employing both multiple transmit and multiple receive antennas, similar trends are observable, although using two or four receive antennas has the potential of mitigating the associated acquisition performance degradation imposed by the low per-branch $E_{c} / I_{o}$ values associated with the employment of multiple transmitters. Fig.5 and Fig. 6 characterise the achievable MAT versus SINR per chip performance of post-initial acquisition. The results are parameterised by both the number of transmit antennas for $P=1,2$ as well as 4 and by the number of receive antennas for $R=1,2$ as well as 4 for both the SDSS (Fig.5) and for the DDSS schemes (Fig.6), respectively. Even though the optimised coherent summation intervals determined for the sake of obtaining the best possible MAT performance are quite different, as the number of transmit antennas is decreased, all the curves seen in both Fig.5 and Fig.6 indicate an improved MAT peformance, as we observed in the case of intial acquisition in both Fig. 2 and Fig.3. This trend explicitly indicates that the DDSS scheme also degrades the achievable MAT performance as a consequence of the low per-antenna power imposed by employing multiple transmit antennas for the sake of attaining a transmit diversity gain. However, the MAT peformance degradation imposed is less severe than that of the SDSS scheme. To interpret the above results a little further, a low level of per-branch received signal strength would lead to a low acquisition performance, despite achieving a high transmit diversity gain. In other words, a high transmit diversity order effectively results in an acquisition performance loss, as a consequence of the insufficiently high signal strength per transmit antenna branch.

\section{CONCLUSION}

In this paper, we analysed the multiple antenna aided transmit/receive diversity effects on the performance of both initial and post-initial acquisition schemes in the inter-cell synchronous CDMA downlink. The probabilities of correct detection and false alarm have been derived analytically and numerical results were provided in terms of the attainable MAT performance. Ironically, our findings suggest that increasing the number of transmit antennas in a MIMO-aided CDMA system results in combining the lowenergy, noise-contaminated signals of the transmit antennas, which ultimately increases the MAT by an order of magnitude, when the SINR is relatively low. This phenomenon has a detrimental effect on the performance of Rake receiver based synchronisation, when the perfectly synchronised system is capable of attaining its target bit error rate performance at reduced SINR values, as a benefit of employing multiple transmit antennas. Therefore our future research will be aimed at specifically designing iterative turbo acquisition schemes for MIMO systems [3], [12].

\section{REFERENCES}

[1] D. Gesbert, M. Shafi, D.S. Shiu, P.J. Smith, and A. Naguib, From Theory to Practice: An Overview of MIMO Space-Time Coded Wireless Systems, IEEE Journal on Selected Areas in Communications, vol. 21, NO.3, Issue 3, 2003, pp281-302.

[2] B-G Lee, B-H Kim, Scrambling Techniques For CDMA Communications, Chapter 2 and 3, Kluwer Academic Publishers, 2001.

[3] L. Hanzo, L-L Yang, E-L Kuan, K. Yen, Single- and MultiCarrier DS-CDMA, IV Multi-Carrier CDMA, Chapter 21, Initial Synchronization of DS-CDMA and MC-CDMA Systems, Wiley, 2003.

[4] S. Glisic, M.D. Katz, Modeling of the Code Acquisition Process for Rake Receivers in CDMA Wireless Networks with Multipath and Transmitter Diversity, IEEE Journal on Selected Areas in Communications, vol. 19, NO.1, Issue 1, 2001, pp21-32.

[5] S.H. Won and Y.J. Kim, Performance Analysis of Multi-path Searcher for Mobile Station in W-CDMA System Employing Transmit Diversity, Electronics Letters, vol. 39, Issue 1, 2003, pp137-139.

[6] A.J. Viterbi, CDMA: Principles of Spread Spectrum Communication, Chapter 3, Addison-Wesley, 1995.

[7] 3GPP TS 25.101 V4.11.0, User Equipment (UE) radio transmission and reception (FDD)

[8] H-R Park and B-J Kang, On Serial Search Code Acquisistion for Direct-Sequence Spread Spectrum System: An Applicaion to IS95 CDMA System, Vehicular Technology Conference, 1995, vol. 1, 25-28 July 1995, pp291-295.

[9] J-C Lin, Differentially coherent PN code acquisition with full-period correlation in chip-synchronous DS/SS receivers, IEEE Transactions on Communications, vol. 50, NO.5, Issue 5, 2002, pp698-702.

[10] J.G. Proakis, Digital Communications, 3rd ed. Chapter 2, McGrawHill, 1995

[11] H.R. Park, Performance Analysis of a Double-Dwell Serial Search Technique for Cellular CDMA Networks in the Case of Multiple Pilot Signals, IEEE Transactions on Vehicular Technology, vol. 48, NO.6, Issue 6, 1999, pp1819-1830.

[12] L-L Yang, L. Hanzo, Acquisition of m-sequences using recursive soft sequential estimation, IEEE Transactions on Communications, vol. 52, Issue 2, 2004, pp199-204.

TABLE I

MAXIMUM SINR DEGRADATION INFLICTED BY BOTH THE DOPPLER SHIFT AND A 1000 HZ FREQUENCY MISMATCH IN CONJUNCTION WITH THE COHERENT INTEGRATION INTERVAL OF $N$ CHIP DURATIONS AT A CARRIER FREQUENCY OF $1.9 \mathrm{GHZ}$

\begin{tabular}{|l|l|l|l|l|l|}
\hline $\mathrm{N}($ Chips $)$ & 64 & 128 & 256 & 384 & 512 \\
\hline Degradation $(\mathrm{dB})$ & 0.061 & 0.2449 & 0.9969 & 2.3144 & 4.3213 \\
\hline
\end{tabular}

TABLE II

MAXIMUM SINR DEGRADATION INFLICTED BY BOTH THE DOPPLER SHIFT AND A 200HZ FREQUENCY MISMATCH IN CONJUNCTION WITH THE COHERENT INTEGRATION INTERVAL OF $N$ CHIP DURATIONS AT A CARRIER FREQUENCY OF $1.9 \mathrm{GHz}$

\begin{tabular}{|l|l|l|l|l|l|l|}
\hline $\mathrm{N}($ Chips) & 128 & 256 & 384 & 512 & 640 & 768 \\
\hline Degradation $(\mathrm{dB})$ & 0.032 & 0.128 & 0.289 & 0.5159 & 0.812 & 1.179 \\
\hline
\end{tabular}

\section{ACKNOWLEDGMENT}

The financial support of the EPSRC, UK and that of the European Union under the auspices of the Phoenix and Newcom projects is gratefully acknowledged. 
TABLE III

OPTIMISED LENGTH OF COHERENT SUMMATION OF DETECTOR OUTPUTS INVOLVED FOR THE SAKE OF ANALYSING SDSS IN POST-INITIAL ACQUISITION

\begin{tabular}{|l|l|l|l|l|l|}
\hline $\begin{array}{l}\text { Transmit } \\
\text { /Receive }\end{array}$ & $\begin{array}{l}\text { Length } \\
\text { (Chips) }\end{array}$ & $\begin{array}{l}\text { Transmit } \\
\text { /Receive }\end{array}$ & $\begin{array}{l}\text { Length } \\
\text { (Chips) }\end{array}$ & $\begin{array}{l}\text { Transmit } \\
\text { /Receive }\end{array}$ & $\begin{array}{l}\text { Length } \\
\text { (Chips) }\end{array}$ \\
\hline P1R1 & 512 & P1R2 & 384 & P1R4 & 256(128) \\
\hline P2R1 & 512 & P2R2 & 384 & P2R4 & $256(384)$ \\
\hline P4R1 & 640 & P4R2 & 512 & P4R4 & 384 \\
\hline
\end{tabular}

TABLE IV

OPTIMISED LENGTH OF COHERENT SUMMATION OF DETECTOR OUTPUTS INVOLVED IN VERIFICATION MODE FOR THE SAKE OF ANALYSING DDSS IN POST-INITIAL ACQUISITION

\begin{tabular}{|l|l|l|l|l|l|}
\hline $\begin{array}{l}\text { Transmit } \\
\text { /Receive }\end{array}$ & $\begin{array}{l}\text { Length } \\
\text { (Chips) }\end{array}$ & $\begin{array}{l}\text { Transmit } \\
\text { /Receive }\end{array}$ & $\begin{array}{l}\text { Length } \\
\text { (Chips) }\end{array}$ & $\begin{array}{l}\text { Transmit } \\
\text { /Receive }\end{array}$ & $\begin{array}{l}\text { Length } \\
\text { (Chips) }\end{array}$ \\
\hline P1R1 & 384 & P1R2 & $384(512)$ & P1R4 & $256(384)$ \\
\hline P2R1 & 640 & P2R2 & $512(640)$ & P2R4 & 384 \\
\hline P4R1 & 768 & P4R2 & $640(768)$ & P4R4 & 512 \\
\hline
\end{tabular}

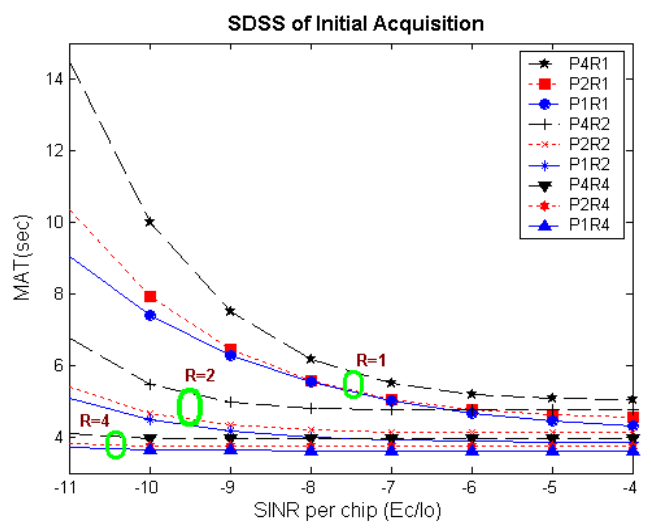

Fig. 2. MAT versus SINR per chip performance of the initial acquisition system for SDSS parameterised with both the number of transmit and receive antennas.

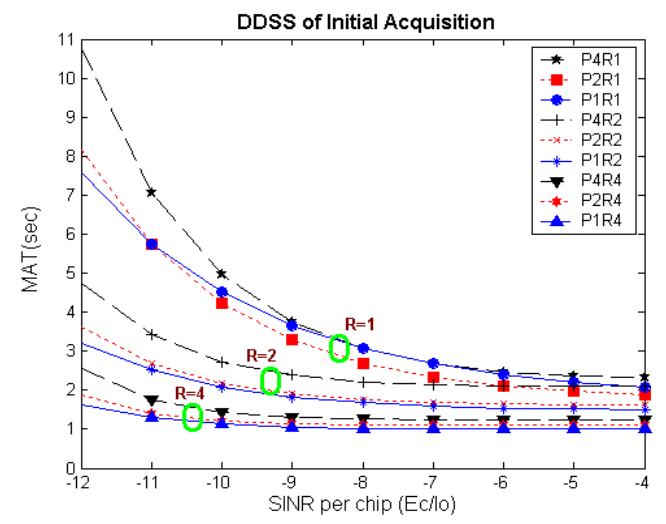

Fig. 3. MAT versus SINR per chip performance of the initial acquisition system for DDSS parameterised with both the number of transmit and receive antennas.

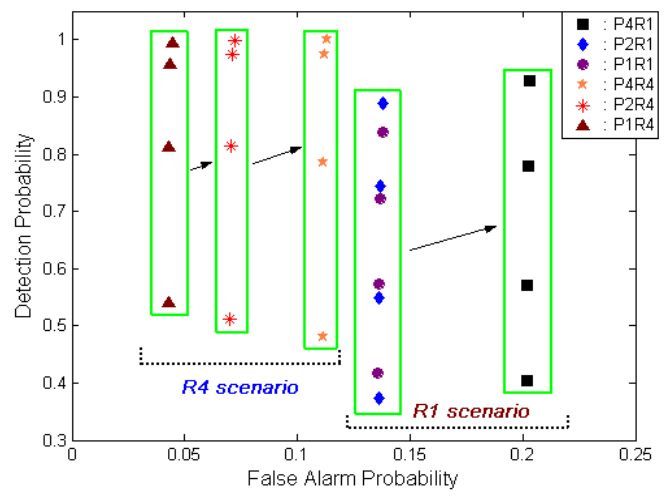

Fig. 4. Operating ranges in the search mode of the initial acquisition scheme for the sake of obtaining the best possible MAT performance. The four vertically stacked points seen in the figure correspond to $E_{c} / I_{O}=-4,-7$, -10 and $-13 \mathrm{~dB}$, respectively, from the top to the bottom.

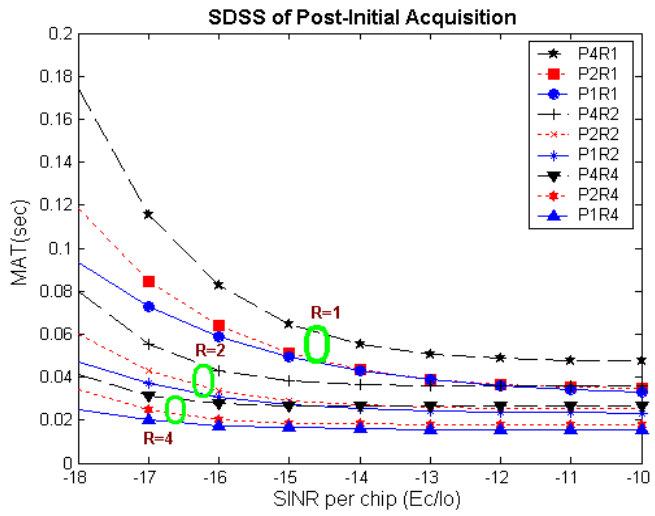

Fig. 5. MAT versus SINR per chip performance of the post-initial acquisition system for SDSS parameterised with both the number of transmit and receive antennas.

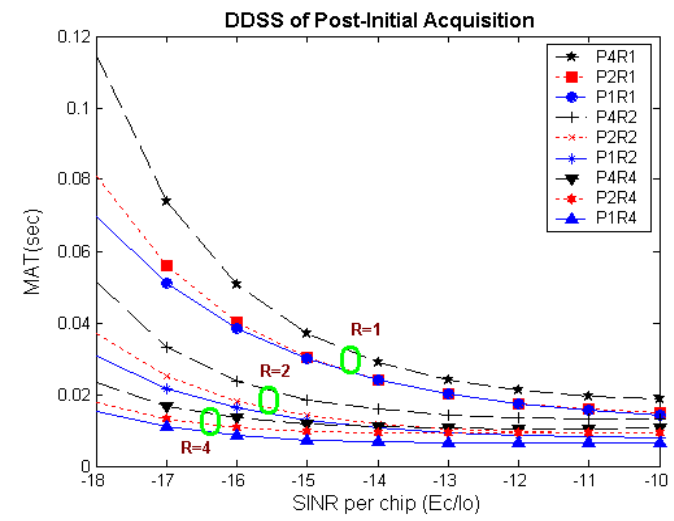

Fig. 6. MAT versus SINR per chip performance of the post-initial acquisition system for DDSS parameterised with both the number of transmit and receive antennas. 